\title{
COMPUTING THE MASLOV INDEX FOR LARGE SYSTEMS
}

\author{
MARGARET BECK AND SIMON J. A. MALHAM
}

(Communicated by Ken Ono)

\begin{abstract}
We address the problem of computing the Maslov index for large linear symplectic systems on the real line. The Maslov index measures the signed intersections (with a given reference plane) of a path of Lagrangian planes. The natural chart parameterization for the Grassmannian of Lagrangian planes is the space of real symmetric matrices. Linear system evolution induces a Riccati evolution in the chart. For large order systems this is a practical approach as the computational complexity is quadratic in the order. The Riccati solutions, however, also exhibit singularites (which are traversed by changing charts). Our new results involve characterizing these Riccati singularities and two trace formulae for the Maslov index as follows. First, we show that the number of singular eigenvalues of the symmetric chart representation equals the dimension of intersection with the reference plane. Second, the Cayley map is a diffeomorphism from the space of real symmetric matrices to the manifold of unitary symmetric matrices. We show the logarithm of the Cayley map equals the arctan map (modulo 2i) and its trace measures the angle of the Langrangian plane to the reference plane. Third, the Riccati flow under the Cayley map induces a flow in the manifold of unitary symmetric matrices. Using the natural unitary action on this manifold, we pullback the flow to the unitary Lie algebra and monitor its trace. This avoids singularities, and is a natural robust procedure. We demonstrate the effectiveness of these approaches by applying them to a large eigenvalue problem. We also discuss the extension of the Maslov index to the infinite dimensional case.
\end{abstract}

\section{INTRODUCTION}

Our goal is to compute the Maslov index for non-autonomous, symplectic, linear differential operators of the form

$$
\partial-A
$$

with domain the real line $\mathbb{R}$. Here $A: \mathbb{R} \rightarrow \mathfrak{s p}\left(\mathbb{R}^{2 n}\right)$, the symplectic Lie algebra of real $2 n \times 2 n$ matrices. We are particularly interested in the case when $n$ is large. We assume that the matrix $A$ is constant in the far-field limits of the domain $\mathbb{R}$ (the two limits need not be the same). The Maslov index is an integer valued topological invariant that measures the following occurences. Consider the set of (necessarily) $n$ solutions that decay exponentially fast, say, to the left far-field. These solutions constitute a path of frames, indeed a path in the Stiefel manifold of symplectic frames $\mathrm{V}\left(\mathbb{R}^{2 n}\right)$. Each frame spans a plane in the manifold of Lagrangian planes $\Lambda\left(\mathbb{R}^{2 n}\right)$ known as the Lagrangian Grassmannian. The fundamental group of $\Lambda\left(\mathbb{R}^{2 n}\right)$ is $\pi_{1}\left(\Lambda\left(\mathbb{R}^{2 n}\right)\right)=\mathbb{Z}$. We evolve the Lagrangian plane from the left far-field towards

Received by the editors November 5, 2013.

2010 Mathematics Subject Classification. Primary 35P05.

The work of the first author was partially supported by NSF DMS 1007450 and a Sloan Fellowship. 
the right far-field and count the number of intersections this Lagrangian plane path has with a given reference plane. The dimension of each intersection generates the multiplicity contribution to the Maslov index, and the direction of passage generates its sign contribution. There are various equivalent ways to formulate the definition of the Maslov index more precisely, and we refer to Furutani [11 for more details. This picture can be succinctly summarized in terms of cohomological classes of paths; see Arnol'd [3].

One of the main motivations for studying operators of the form $\partial-A$ is that they arise in the context of eigenvalue problems associated with the stability of solutions of travelling waves. For example, suppose one is interested in determining the qualitative behaviour of solutions of the reaction-diffusion system

$$
\partial_{t} U=B \partial_{x}^{2} U+F(U)
$$

where $U: \mathbb{R} \times \mathbb{R}^{+} \rightarrow \mathbb{R}^{n}$ and $B$ is a diagonal matrix of positive diffusion coefficients. A key step is to determine the existence of any stationary solutions, say $U_{*}(x)$, and their stability properties. The reason is that stable solutions attract nearby data and therefore provide a qualitative description of the types of behaviours that one would expect to observe as the system evolves. In order to determine if $U_{*}$ is stable, one can linearize the reaction-diffusion system about $U_{*}$ to obtain

$$
H:=B \partial_{x}^{2}+V(x),
$$

where $V(x):=\mathrm{d} F\left(U_{*}(x)\right)$ is the potential. In general, a necessary condition for $U_{*}$ to be stable is that the spectrum of this linear operator, in an appropriate function space such as $L^{2}(\mathbb{R})$, be contained in the closed left half plane. The most difficult part of the spectrum to analyze is typically the point spectrum, or eigenvalues, which can be determined by studying

$$
(H-\lambda) U=O .
$$

We assume $V$ is selfadjoint and set $q:=(\operatorname{Re} U, \operatorname{Im} U)^{\mathrm{T}}$. Then if we set $p:=\partial_{x} q$ we can rewrite this eigenvalue problem as a first order system (hereafter $\partial:=\partial_{x}$ )

$$
(\partial-A)\left(\begin{array}{l}
q \\
p
\end{array}\right)=O
$$

where $A=A(x, \lambda)$ is the corresponding symplectic coefficient matrix. Eigenvalues then correspond to values of $\lambda$ for which solutions $(q, p)^{\mathrm{T}}$ exist that decay to zero as $x \rightarrow \pm \infty$. This asymptotic behaviour is equivalent to requiring that the solution lie in the intersection of two particular Lagrangian subspaces, namely the stable and unstable manifolds associated with $x=+\infty$ and $x=-\infty$, respectively. Thus, being able to determine such intersections, via the Maslov index (and a matrix Riccati equation as we will see), would provide insight into the stability of $U_{*}$. Such a framework is not limited to reaction-diffusion equations. It also applies to a variety of other examples, including higher-order equations such as the dispersive KdV equation. See Section 6 and examples in Chardard et al. [7].

There is a large body of existing literature on determining the spectral properties of the types of linear operators mentioned above. Perhaps the most widely used tool is the Evans function (Alexander, Gardner and Jones [1, Sandstede 24], Deng and Nii [10], Sandstede and Oh [25]), which is constructed for systems governed by operators of the form $\partial-A$. The use of the Maslov index in such contexts is relatively recent; see Jones [15], Chardard et al. 7], Deng and Jones [9], Jones et al. 14. A limitation of the majority of these results is that they apply only to 
problems in one spatial dimension. As a result, there has been recent interest in developing techniques that apply to arbitrary spatial dimensions. Notable steps in this direction include Deng and Nii [10] and Sandstede and Oh [25], involving the Evans function, and Deng and Jones [9], involving the Maslov index. However, there has not yet been an application of these abstract results to concrete examples, where the stability of a specific stationary solution is actually computed. One potential consequence of this current work is to provide a mechanism to do just that. Much of the theoretical framework herein in principle applies to arbitrary symplectic Hilbert spaces, such as those of the multidimensional case considered in Deng and Jones [9]. Indications of this are given in Section 7

Intuitively, the Maslov index can provide information about stability in the following way. Consider the reaction-diffusion system above with $U \in \mathbb{R}$. This is just a Sturm-Liouville problem, and it can be recast in terms of Prüfer coordinates, which are polar coordinates for $(q, p)^{\mathrm{T}}$. One can then determine the dynamics of the phase and prove a so-called oscillation theorem. This says that, as the eigenvalue parameter is increased, the amount of oscillation in the phase of the corresponding solution decreases. One thus obtains a result concerning the sequence of eigenvalues $\lambda_{0}>\lambda_{1}>\ldots$ and corresponding eigenfunctions $U_{0}, U_{1}, \ldots$, which says that $U_{k}$ has exactly $k$ zeros. This is particularly useful when $\partial-A$ arises from a system such as the reaction-diffuson system above by linearizing about the stationary solution $U_{*}$. In this case, $\partial_{x} U_{*}$ is an eigenfunction associated with the eigenvalue $\lambda=0$. If, for example, $U_{*}$ is a pulse, then $\partial_{x} U_{*}$ has exactly one zero. Hence, there is exactly one eigenvalue with positive real part and the wave is spectrally unstable. Similarly, if $U_{*}$ is a front, then the linearization has no positive eigenvalues and the wave is spectrally stable. The Maslov index can be thought of as a higher-dimensional analogue of this oscillation index that applies to a more general class of equations. The winding, or oscillation, in the general case comes from the fact that the fundamental group of the space of Lagrangian planes is $\mathbb{Z}$. Note that the Maslov index and the Evans function provide stability information in a fundamentally different way. The latter is an analytic function whose zeros correspond to eigenvalues, and thus in principle one can locate the eigenvalues by locating its zeros. The former, however, allows one to count, but not locate, the eigenvalues.

Computing the Maslov index associated with $\partial-A$ will in general involve numerical integration. Recently Chardard et al. 7] demonstrated how to numerically compute the Malsov index for solitary waves. To circumvent the inherent numerical instabilities associated with computing the path of symplectic frames where the frame vectors have distinct exponential growth rates, Chardard et al. lifted the path in the Stiefel manifold of symplectic frames $\mathrm{V}\left(\mathbb{R}^{2 n}\right)$ to a path in the symplectic exterior algebra $\bigwedge^{n} \mathrm{~V}\left(\mathbb{R}^{2 n}\right)$. This path has a single exponential growth rate that can be scaled out and can be robustly numerically computed. The number of parameters required to represent $\bigwedge^{n} \mathrm{~V}\left(\mathbb{R}^{2 n}\right)$ is $2 n$ choose $n$, which grows exponentially with $n$. The Plücker relations between the parameters can be used to reduce the dimension of the system. A natural resolution is to apply the ortho-symplectic integration proposed by Chardard et al. or indeed a symplectic version of the continuous orthogonalization method proposed by Humpherys and Zumbrun [13.

Here we pursue another natural approach which utilizes the principle fibre bundle structure $\mathrm{GL}\left(\mathbb{R}^{n}\right) \rightarrow \mathrm{V}\left(\mathbb{R}^{2 n}\right) \rightarrow \Lambda\left(\mathbb{R}^{2 n}\right)$. Indeed we project $\mathrm{V}\left(\mathbb{R}^{2 n}\right)$ onto the Lagrangian Grassmannian $\Lambda\left(\mathbb{R}^{2 n}\right)$ by equivalencing by all general linear real rank $n$ transformations $\operatorname{GL}\left(\mathbb{R}^{n}\right)$. Following Ledoux et al. [18, the flow governed by $\partial-A$ 
on $\mathrm{V}\left(\mathbb{R}^{2 n}\right)$ induces a Riccati flow in a given chart and top cell $\Lambda^{0}\left(\mathbb{R}^{2 n}\right)$ of the Lagrangian Grassmannian $\Lambda\left(\mathbb{R}^{2 n}\right)$. The Riccati flow exhibits singularities but these just indicate the chart chosen $\Lambda^{0}\left(\mathbb{R}^{2 n}\right)$ is no longer the best representative chart. However, crucially, when integrating Riccati equations in the context of eigenvalue problems as described above, indeed for non-selfadjoint problems, Ledoux recognized that the location (in the complex spectral parameter plane) of singularities in the components of the Riccati solution converged to eigenvalues in the far-field limit. See Ledoux and Malham [16] and Aljasser [2] for more details. Our results here clarify and confirm these observations in the symplectic context.

Note that the Lagrangian Grassmannian chart $\Lambda^{0}\left(\mathbb{R}^{2 n}\right)$ is isomorphic to vector space of real symmetric matrices, and the Cayley map is a diffeomorphism from the space of real symmetric matrices to the manifold of unitary symmetric nonsingular matrices (see Section 2). From Arnol'd [3] we know the logarithm of the determinant of the Cayley map measures the angle of the Lagrangian plane to the reference plane. What we prove that is new is:

(1) The number of singular eigenvalues in the Riccati flow on $\Lambda^{0}\left(\mathbb{R}^{2 n}\right)$ equals the dimension of intersection with the reference plane, which equals the unsigned integer jump in the Maslov index.

(2) The logarithm of the Cayley map equals the arctan map (modulo 2i) and its trace computes the angle between the evolving Langrangian and reference planes. Hence in principle we could push forward the Riccati flow under the arctan map and monitor its trace.

(3) The Riccati flow under the Cayley map induces a flow in the manifold of unitary symmetric non-singular matrices. Using the natural unitary action on this manifold, we pullback the flow to the unitary Lie algebra and monitor its trace. This avoids singularities, and is a natural robust procedure for monitoring the Maslov index.

Our paper is organised as follows. In Section 2 we fix our notation, define the Lagrangian Grassmannian and introduce known results that we will need. We briefly discuss in Section 3 the well-known method of changing coordinates to standardize the reference Lagrangian plane we seek intersections with. In Section 4 we derive the Riccati flow prescribing the evolution by $\partial-A$ of a Lagrangian plane and establish the connection between singularities in the flow and intersections with the reference plane. We provide the details of how to pull back the flow to the unitary Lie algebra in Section 5 . We compute the Maslov index for a large (high order) eigenvalue problem in Section [6 and demonstrate the computational advantages of the methods we have derived/propose. Finally in Section 7 we discuss the extension to the infinite dimensional case.

\section{Lagrangian Grassmannian}

We introduce the objects of our study and fix our notion as follows. We denote by $\operatorname{Sp}\left(\mathbb{R}^{n}\right), \mathrm{GL}\left(\mathbb{C}^{n}\right), \mathrm{O}\left(\mathbb{R}^{n}\right)$ and $\mathrm{U}\left(\mathbb{C}^{n}\right)$, respectively, the usual symplectic, general linear, orthogonal and unitary Lie groups of rank $n$ matrices over the fields indicated. We use $\mathfrak{s p}\left(\mathbb{R}^{n}\right)$ and $\mathfrak{u}\left(\mathbb{C}^{n}\right)$ to denote the corresponding symplectic and unitary Lie algebras. We denote by:

- $\mathrm{V}\left(\mathbb{R}^{2 n}\right)$, the Stiefel manifold of symplectic $n$-frames in $\mathbb{R}^{2 n}$;

- $\Lambda\left(\mathbb{R}^{2 n}\right)$, the Lagrangian Grassmann manifold of $n$-planes in $\mathbb{R}^{2 n}$;

- $\mathrm{U}_{\text {sym }}\left(\mathbb{C}^{n}\right)$, the manifold of unitary symmetric non-singular matrices in $\mathbb{C}^{n}$. 
Our subsequent analysis mainly involves these standard Lie groups and homogeneous manifolds. Some of the important relations between them are summarized in the following diagram:

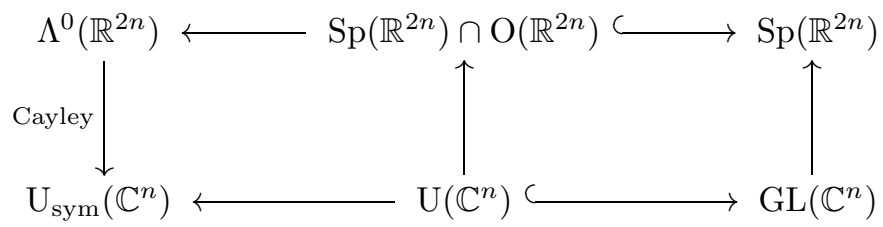

Here the vertical lift maps are isomorphisms and all the horizontal maps are embeddings and projections as indicated. The relations between the four groups on the right are given by natural isomorphic liftings of the respective complex groups (bottom) to their real counterparts (top), combined with the isomorphism $\mathrm{U}\left(\mathbb{C}^{n}\right) \cong \mathrm{GL}\left(\mathbb{C}^{n}\right) \cap \mathrm{O}\left(\mathbb{R}^{2 n}\right)$. The bottom left projection results from the fibering

$$
\mathrm{O}\left(\mathbb{R}^{n}\right) \rightarrow \mathrm{U}\left(\mathbb{C}^{n}\right) \rightarrow \mathrm{U}_{\text {sym }}\left(\mathbb{C}^{n}\right)
$$

The leftmost diffeomorphism, the Cayley transform, is explained as follows - more details can be found in Arnol'd [3] and de Gosson [8]. The Lagrangian Grassmannian is equivalently defined via the fibering

$$
\mathrm{GL}\left(\mathbb{R}^{n}\right) \rightarrow \mathrm{V}\left(\mathbb{R}^{2 n}\right) \rightarrow \Lambda\left(\mathbb{R}^{2 n}\right) .
$$

Suppose a symplectic frame in $\mathrm{V}\left(\mathbb{R}^{2 n}\right)$ is represented by a $2 n \times n$ matrix. Charts that cover $\Lambda\left(\mathbb{R}^{2 n}\right)$ are represented by the set of $2 n \times n$ matrices with distinct $n \times n$ submatrices given by the identity. There are $2 n$ choose $n$ charts. For example if $q$ and $p$ are square $n \times n$ matrices forming a frame in $\mathrm{V}\left(\mathbb{R}^{2 n}\right)$ we can choose a chart on $\Lambda\left(\mathbb{R}^{2 n}\right)$ by projecting as follows:

$$
\left(\begin{array}{l}
q \\
p
\end{array}\right) \mapsto\left(\begin{array}{c}
\text { id } \\
s
\end{array}\right)
$$

where $s: q \mapsto p$ is necessarily symmetric due to symplectic condition on the frame. We observe the Lagrangian Grassmannian is thus $\frac{1}{2} n(n+1)$ dimensional. The set of Lagrangian planes $\Lambda^{0}\left(\mathbb{R}^{2 n}\right)$ represented in this chart are dense in $\Lambda\left(\mathbb{R}^{2 n}\right)$, and we shall denote $\Lambda^{0}\left(\mathbb{R}^{2 n}\right)$ as the top Schubert cell in $\Lambda\left(\mathbb{R}^{2 n}\right)$. The Lagrangian Grassmannian $\Lambda\left(\mathbb{R}^{2 n}\right)$ is the disjoint union of all the Schubert cells (more on this presently). As we have already indicated, $\Lambda^{0}\left(\mathbb{R}^{2 n}\right)$ is diffeomorphic to the linear space of real symmetric matrices $\mathrm{D}\left(\mathbb{R}^{n}\right)$ of order $n$. The Cayley transform Cay: $\mathrm{D}\left(\mathbb{R}^{n}\right) \rightarrow \mathrm{U}_{\text {sym }}\left(\mathbb{C}^{n}\right)$ given by

$$
\text { Cay }: s \mapsto \frac{\mathrm{id}-\mathrm{i} s}{\mathrm{id}+\mathrm{i} s}
$$

is a diffeomorphism and plays an important role in our main results. From $\mathrm{Arnol}^{\prime} \mathrm{d}\left[3\right.$ there is a natural map $\operatorname{Det}^{2}: \Lambda\left(\mathbb{R}^{2 n}\right) \rightarrow \mathbb{S}^{1}$ given by

$$
\operatorname{Det}^{2}:=\operatorname{det} \circ \text { Cay. }
$$

We deduce the fundamental group $\pi_{1}\left(\Lambda\left(\mathbb{R}^{2 n}\right)\right) \cong \mathbb{Z}$.

Remark 2.1. In coordinates, for $s \in \Lambda^{0}\left(\mathbb{R}^{2 n}\right)$ the map $\operatorname{Det}^{2}$ is straightforward via the above decomposition. However it is well defined for any plane in $\Lambda\left(\mathbb{R}^{2 n}\right) \backslash \Lambda^{0}\left(\mathbb{R}^{2 n}\right)$, i.e. in any lower Schubert cell, as we see in detail in Section 4 


\section{Standard Reference Plane And total frame matrix}

Assume we have a path in the Stiefel manifold of symplectic frames $V\left(\mathbb{R}^{2 n}\right)$ generated by the solution flow of the non-autonomous linear differential system on the real line $\mathbb{R}$,

$$
(\partial-A)\left(\begin{array}{l}
q \\
p
\end{array}\right)=O
$$

Here $q$ and $p$ are real $n \times n$ matrices and $A: \mathbb{R} \rightarrow \mathfrak{s p}\left(\mathbb{R}^{2 n}\right)$ has the block form

$$
A:=\left(\begin{array}{ll}
a & b \\
c & d
\end{array}\right)
$$

where $a, b, c$ and $d$ are real $n \times n$ matrices with $b$ and $c$ symmetric and $a=-d^{\mathrm{T}}$. The path of symplectic frames over $\mathbb{R}$ induces a path of Lagrangian planes (spanning each frame). We wish to determine the number and type of intersections of the path of Lagrangian planes with a reference plane. The occurence and dimension of their intersection is measured by the drop in rank of the $2 n \times 2 n$ matrix

$$
\left(\begin{array}{ll}
q & q_{0} \\
p & p_{0}
\end{array}\right)
$$

where the left $2 n \times n$ block represents our evolutionary symplectic frame in $V\left(\mathbb{R}^{2 n}\right)$ and the right $2 n \times n$ block represents the reference plane in $V\left(\mathbb{R}^{2 n}\right)$. Since the reference plane is fixed, it is natural to seek a change of coordinates to carry the reference plane to the standard reference plane with $q_{0}=O$ and $p_{0}=$ id. A linear rank $2 n$ transformation of coordinates that achieves this is

$$
\left(\begin{array}{cc}
q^{\prime} & O \\
p^{\prime} & \mathrm{id}
\end{array}\right)=\left(\begin{array}{cc}
\mathrm{id} & -q_{0} p_{0}^{-1} \\
O & p_{0}^{-1}
\end{array}\right)\left(\begin{array}{cc}
q & q_{0} \\
p & p_{0}
\end{array}\right)
$$

where $q^{\prime}:=q-q_{0} p_{0}^{-1} p$ and $p^{\prime}:=p_{0}^{-1} p$. This assumes that $p_{0}$ is non-singular for the reference plane; it is not difficult to adapt our subsequent arguments if this is not the case. Note that rank is conserved under this linear transformation.

Definition 3.1 (Total frame matrix). This is the $2 n \times 2 n$ matrix given by

$$
\left(\begin{array}{ll}
q^{\prime} & O \\
p^{\prime} & \text { id }
\end{array}\right)
$$

Its loss of rank measures the occurence and dimension of the intesection of the plane spanned by the frame $\left(q^{\prime}, p^{\prime}\right)^{\mathrm{T}} \in V\left(\mathbb{R}^{2 n}\right)$ with the standard reference plane, or equivalently, the plane spanned by $(q, p)^{\mathrm{T}} \in V\left(\mathbb{R}^{2 n}\right)$ with $\left(q_{0}, p_{0}\right)^{\mathrm{T}} \in V\left(\mathbb{R}^{2 n}\right)$.

Remark 3.2. Since $(q, p)$ satisfy the linear system generated by $\partial-A$, then $\left(q^{\prime}, p^{\prime}\right)$ satisfy an analogous transformed linear system.

Remark 3.3. Note that, as they represent a solution frame in $\mathrm{V}\left(\mathbb{R}^{2 n}\right)$ of a linear system, $q^{\prime}$ and $p^{\prime}$ (or $q$ and $p$ ) are finite matrices whose components can only become singular in either far-field limit.

Henceforth we will drop 'primes' and assume $q^{\prime} \rightarrow q$ and $p^{\prime} \rightarrow p$. 


\section{Riccati FLOW IN THE CHART}

Recall the Lagrangian Grassmannian chart we denoted as the top cell $\Lambda^{0}\left(\mathbb{R}^{2 n}\right)$ in Section 2. We derive the Riccati flow in $\Lambda^{0}\left(\mathbb{R}^{2 n}\right)$ generated by the linear symplectic flow in $\mathrm{V}\left(\mathbb{R}^{2 n}\right)$. Define $s \in \mathrm{D}\left(\mathbb{R}^{n}\right)$ as the real symmetric map $s: q \mapsto p$. Note $q$ and $p$ satisfy the equations $\partial q=(a+b s) q$ and $\partial p=(c+d s) q$. Using $\partial(s q)=\partial s q+s \partial q$ we know $\partial s q=\partial p-s \partial q$. Direct substitution reveals $\partial s q=c q+d s q-s(a q+b s q)$. Hence we see that $s$ satisfies the Riccati equation

$$
\partial s=c+d s-s(a+b s) .
$$

Thus $s \in \mathrm{D}\left(\mathbb{R}^{n}\right)$ prescribes the evolution of the Lagrangian planes which can be represented in the top cell $\Lambda^{0}\left(\mathbb{R}^{2 n}\right)$. Recall that $\Lambda^{0}\left(\mathbb{R}^{2 n}\right)$ is dense in $\Lambda\left(\mathbb{R}^{2 n}\right)$ and the set of Lagrangian planes in $\Lambda\left(\mathbb{R}^{2 n}\right) \backslash \Lambda^{0}\left(\mathbb{R}^{2 n}\right)$ are all those respresented in the lower dimensional Schubert cells. Components in the Riccati solution $s$ can become singular in finite time and this is the signature that the Lagrangian plane is no longer representable in $\Lambda^{0}\left(\mathbb{R}^{2 n}\right)$ and moves into a lower Schubert cell. In turn this induces a drop in rank in $q$ and thus also the total frame matrix.

More precisely, let the real eigenvalues of $s$ be $\mu_{i}, i=1, \ldots, n$. Imagine we evolve our frame $(q, p)^{\mathrm{T}} \in V\left(\mathbb{R}^{2 n}\right)$ under $\partial-A$ from one far-field to the other. For all $x \in \mathbb{R}$ the solution components $q=q(x)$ and $p=p(x)$ are well defined, bounded and the frame $(q(x), p(x))^{\mathrm{T}}$ has rank $n$. Alongside this frame, we evolve $s: \mathbb{R} \rightarrow \Lambda^{0}\left(\mathbb{R}^{2 n}\right)$, i.e. $s=p q^{-1}$, under its natural Riccati evolution. Note that $s=s(x)$ is well defined on $\operatorname{Rg} q(x)$, the range of the operator $q(x)$. We say that $\mu_{i}=\mu_{i}(x)$ is a singular eigenvalue of $s=s(x)$ at $x=x_{0}$ if $\lim _{x \rightarrow x_{0}}\left|\mu_{i}(x)\right|=\infty$.

Theorem 4.1 (Equivalent Maslov index contributions). When the Lagrangian plane intersects the standard reference plane, the following integer valued quantities are equal (we do not concern ourselves with the direction of crossing here):

(1) unsigned integer jump value in the Maslov index;

(2) rank loss in the matrix q;

(3) rank loss in the total frame matrix;

(4) dimension of intersection of Lagrangian and standard reference planes;

(5) number of singular eigenvalues of $s$.

Proof. The first four equivalences can be deduced from results in Arnol'd [3] and de Gosson [8]. Note the rank loss count of de Gosson [8, Lemma 198, Prop. 263] is double ours as we use the total frame matrix rather than a map into $\operatorname{Sp}\left(\mathbb{R}^{2 n}\right)$. Hence our goal is to establish that the rank loss in $q$ equals the number of singular eigenvalues of $s \in \mathrm{D}\left(\mathbb{R}^{n}\right)$. More precisely, we wish to show that for any $x_{0} \in \mathbb{R}$, $s\left(x_{0}\right)$ has exactly $k$ singular eigenvalues if and only if Rank $q\left(x_{0}\right)=n-k$. Recall $q$ and $p$ satisfy the properties $q^{\mathrm{T}} p=p^{\mathrm{T}} q$ and $p q^{\mathrm{T}}=q p^{\mathrm{T}}$, and these imply $s=s(x)$ is symmetric, at least on $\operatorname{Rg} q$. Thus all of the eigenvalues of $s$ are real. Note that $\operatorname{Ker} p \cap \operatorname{Ker} q=\{O\}$ for all $x$, because otherwise there would exist a $v \in \mathbb{R}^{n}$ such that $v \neq O$ and

$$
\left(\begin{array}{c}
q(x) \\
p(x)
\end{array}\right) v=O
$$

which contradicts the fact that the rank of this matrix must be $n$. Next recall that for any square matrix $M$, we have $\mathbb{R}^{n}=\operatorname{Ker} M \oplus \operatorname{Rg} M^{\mathrm{T}}=\operatorname{Ker} M^{\mathrm{T}} \oplus \operatorname{Rg} M$. These facts imply that $q^{-1}: \operatorname{Rg} q \rightarrow \operatorname{Rg} q^{\mathrm{T}}, p: \operatorname{Rg} q^{\mathrm{T}} \rightarrow \operatorname{Rg} q$ and thus $s: \operatorname{Rg} q \rightarrow \operatorname{Rg} q$. 
Suppose $\operatorname{dim} \operatorname{Rg} q=n-k$, which implies that $s$ has at least $n-k$ eigenvalues that are not singular, and therefore at most $k$ singular eigenvalues. Suppose there is an additional one, which implies that $\left.s\right|_{\text {Ker } q^{\mathrm{T}}}$ has an eigenvalue that is not singular: $s\left(x_{0}\right) v\left(x_{0}\right)=\mu\left(x_{0}\right) v\left(x_{0}\right),\left|\mu\left(x_{0}\right)\right|<\infty$, and $v\left(x_{0}\right) \in \operatorname{Ker} q^{\mathrm{T}}$. But we then have

$$
0=q^{\mathrm{T}} v=q^{\mathrm{T}} \mu v=q^{\mathrm{T}} s v=p^{\mathrm{T}} q q^{-1} v=p^{\mathrm{T}} v .
$$

This is a contradiction because $p^{\mathrm{T}}\left(x_{0}\right) v\left(x_{0}\right) \neq O$ if $\operatorname{rank}\left(q^{\mathrm{T}} p^{\mathrm{T}}\right)=n$.

Now suppose $s$ has exactly $k$ singular eigenvalues, and thus $n-k$ non-singular eigenvalues. This implies $\operatorname{dim} \operatorname{Rg} q \leqslant n-k$, and so $\operatorname{dim} \operatorname{Ker} q=\operatorname{dim} \operatorname{Ker} q^{\mathrm{T}} \geqslant k$. If it was strictly greater than $k$, we could again consider the restriction $\left.s\right|_{\operatorname{Ker} q^{\mathrm{T}}}$ and obtain a contradiction as above.

Remark 4.2 (The train). The quantities listed in Theorem 4.1 are equivalent to the dimension of intersection with the train; see Arnol'd [3], Jones [15] or Bose and Jones [5]. They are also equivalent to the drop in dimension from the top to lower Schubert cell. Here, the dimension of a Schubert cell is the number of of real variables required to parameterize it.

Remark 4.3. We assume the points $x_{0} \in \mathbb{R}$ where the eigenvalues of $s$ are singular are isolated. In our applications to spectral problems we will always restrict ourselves to regions of the spectral parameter plane where we know this is true. Moreover, such a degeneracy could be removed via homotopy, and as the Maslov index is invariant under homotopy, this need not affect the count.

Recall the Cayley diffeomorphism Cay: $\mathrm{D}\left(\mathbb{R}^{n}\right) \rightarrow \mathrm{U}_{\text {sym }}\left(\mathbb{C}^{n}\right)$ from Section 2 and that the determinant map composed with this is det Cay: $\mathrm{D}\left(\mathbb{R}^{n}\right) \rightarrow \mathbb{S}^{1}$. Note that $\mathbb{S}^{1} \cong \mathrm{U}\left(\mathbb{C}^{1}\right)$. In coordinates we can thus write

$$
\mathrm{e}^{\mathrm{i} \theta}=\operatorname{det} \text { Cay } s
$$

where $\theta \in[0,2 \pi)$ parameterizes $\mathfrak{u}\left(\mathbb{C}^{1}\right)$ and measures the angle between the evolving Lagrangian and standard reference planes. First, since det $\exp =\exp \operatorname{tr}$ we find

$$
\operatorname{det} \text { Cay }=\exp \operatorname{tr} \log \text { Cay. }
$$

Second, the function $\operatorname{Cay}(s)$ has an expansion in the algebra of operator power series with $\mathbb{C}$-valued coefficients. Hence by the uniqueness of power series we find

$$
\log \text { Cay } s=\log (\mathrm{id}-\mathrm{i} s)-\log (\mathrm{id}+\mathrm{i} s)=2 \mathrm{i} \arctan s .
$$

Putting these two results together we have just proved the following.

Lemma 4.4 (Trace formula). The following maps $\mathrm{D}\left(\mathbb{R}^{n}\right) \rightarrow \mathrm{U}\left(\mathbb{C}^{1}\right)$ are equivalent: $\log \operatorname{det}$ Cay $=2 \mathrm{i} \operatorname{tr} \arctan$.

The angle between the evolving Lagrangian plane and the standard reference plane is thus equivalently given in coordinates by $\theta=-2 \operatorname{tr} \arctan s$.

Remark 4.5. The trace formula implies $\operatorname{tr} \arctan s=\sum_{i=1}^{n} \arctan \mu_{i}$. We can also derive a non-linear equation prescribing the flow of $\arctan s$ directly.

Remark 4.6 (Non-selfadjoint operators). More generally we could consider nonselfadjoint operators $\partial-A$ and thus coefficient matrices $A \in \mathfrak{g l}\left(\mathbb{C}^{n}\right)$, the general linear Lie algebra over $\mathbb{C}$. In this case the natural manifolds of interest are $\mathrm{V}_{k}\left(\mathbb{C}^{n}\right)$, the Stiefel manifold of complex $k$-frames in $\mathbb{C}^{n}$, and $\mathrm{Gr}_{k}\left(\mathbb{C}^{n}\right)$, the Grassmann manifold of $k$-planes in $\mathbb{C}^{n}$. There is a natural fibering $\mathrm{GL}\left(\mathbb{C}^{k}\right) \rightarrow \mathrm{V}_{k}\left(\mathbb{C}^{n}\right) \rightarrow \mathrm{Gr}_{k}\left(\mathbb{C}^{n}\right)$. 
The Grassmannian $\operatorname{Gr}_{k}\left(\mathbb{C}^{n}\right)$ has a Schubert cell structure. The top cell is parameterized by complex $(n-k) \times k$ matrices. We can proceed as for the symplectic case. Suppose $(q, p)^{\mathrm{T}}$ represents a frame in the Stiefel manifold $\mathrm{V}_{k}\left(\mathbb{C}^{n}\right)$, where $q \in \mathbb{C}^{k \times k}$ and $p \in \mathbb{C}^{(n-k) \times k}$. Then $s: q \mapsto p$ parameterizes the top cell and satisfies the Riccati equation $\partial s=c+d s-s(a+b s)$, where $a \in \mathbb{C}^{k \times k}, b \in \mathbb{C}^{k \times(n-k)}, c \in \mathbb{C}^{(n-k) \times k}$ and $d \in \mathbb{C}^{(n-k) \times(n-k)}$ are the blocks of $A$ juxtaposed as for the symplectic case. Ledoux et al. [18, advocated integrating the Riccati equation for $s$ to evaluate the Evans function

$$
\operatorname{det}\left(\begin{array}{cc}
q & q_{0} \\
p & p_{0}
\end{array}\right)
$$

associated with non-selfadjoint eigenvalue problems, where $q_{0}$ and $p_{0}$ represent the far-field data. We can perform the same rank preserving linear transformation as we did in Section 3 to transform the Evans matrix into a form analogous to the total frame matrix; again we reassign $q$ and $p$ as the new transformed solution variables. Then the rank loss in $q$, rank loss in the total frame Evans matrix and dimension of the intersection of unstable and reference planes, are all equivalent. We conjecture they are also equivalent to the number of singular eigenvalues of the matrix $s$.

Remark 4.7 (Superpotentials). If $(q, p)^{\mathrm{T}} \in \mathrm{V}_{k}\left(\mathbb{C}^{n}\right)$, but with $q \in \mathbb{C}^{(n-k) \times k}$ and $p \in \mathbb{C}^{k \times k}$, then the map $r: p \mapsto q$ satisfies the Riccati equation $\partial r=b+a r-r(d+c r)$. The solutions $s$ and $r$ to their respective Riccati equations represent superpotentials, see Bougie et al. 6]. Formally they diagonalize $\partial-A$ in the sense that

$$
\left(\begin{array}{cc}
\text { id } & r \\
s & \text { id }
\end{array}\right)^{-1}(\partial-A)\left(\begin{array}{cc}
\text { id } & r \\
s & \text { id }
\end{array}\right)=\left(\begin{array}{cc}
\partial-(a+b s) & O \\
O & \partial-(d+c r)
\end{array}\right) .
$$

Having diagonalized $\partial-A$ into the block operators $\partial-(a+b s)$ and $\partial-(d+c r)$, we can iterate this procedure, separately diagonalizing $\partial-(a+b s)$ and $\partial-(d+c r)$, and so forth. In principle, provided we can solve the Riccati equations at each level, we can complete a full scalar-block diagonalization of $\partial-A$. We can also Schurtriangularize. If we replace $r$ by $s^{\dagger}$, the transformation operator above becomes unitary and Schur-triangularizes $\partial-A$, which is more akin to the usual factorization procedure in superpotential theory. We can iterate this procedure in an analogous manner to that described for diagonalization.

\section{RICCATI FLOW ON THE SYMMETRIC UNITARY MANIFOLD}

How does the Riccati flow on $\Lambda^{0}\left(\mathbb{R}^{2 n}\right)$ transform under the Cayley diffeomorphism? The answer is a related Riccati flow as follows. Setting

$$
u:=\frac{\mathrm{id}-\mathrm{i} s}{\mathrm{id}+\mathrm{i} s}
$$

and computing the derivative $\partial u$ in terms of $\partial s$ generates the following.

Lemma 5.1. If $s: \mathbb{R} \rightarrow \mathrm{D}\left(\mathbb{R}^{n}\right)$ solves the Riccati equation $\partial s=c+d s-s(a+b s)$, then the corresponding flow $u: \mathbb{R} \rightarrow \mathrm{U}_{\mathrm{sym}}\left(\mathbb{C}^{n}\right)$ is given by

$$
\partial u=C+D u-u\left(D^{*}+C^{*} u\right),
$$

where $C^{*}$ and $D^{*}$ denote the complex conjugates of the $n \times n$ matrices

$$
C:=\frac{1}{2}(a-d-\mathrm{i}(b+c)) \quad \text { and } \quad D:=\frac{1}{2}(a+d+\mathrm{i}(b-c)) .
$$


There is a natural transitive Lie group action $L: \mathrm{U}\left(\mathbb{C}^{n}\right) \times \mathrm{U}_{\mathrm{sym}}\left(\mathbb{C}^{n}\right) \rightarrow \mathrm{U}_{\mathrm{sym}}\left(\mathbb{C}^{n}\right)$, which for all unitary symmetric matrices $u$ and unitary matrices $g$ is given by $L:(g, u) \mapsto g u g^{\mathrm{T}}$. Note this is not an isospectral action. The corresponding Lie algebra action $\ell: \mathfrak{u}\left(\mathbb{C}^{n}\right) \times \mathrm{U}_{\mathrm{sym}}\left(\mathbb{C}^{n}\right) \rightarrow \mathrm{U}_{\mathrm{sym}}\left(\mathbb{C}^{n}\right)$ is given by

$$
\ell:(\xi, u) \mapsto \xi u-u \xi^{*},
$$

for any Lie algebra element $\xi \in \mathfrak{u}\left(\mathbb{C}^{n}\right)$. Recall for $\xi \in \mathfrak{u}\left(\mathbb{C}^{n}\right)$ the complex conjugate transpose $\xi^{\dagger}=-\xi$. We can now establish the following.

Theorem 5.2 (Pullback to the unitary Lie algebra). The Riccati flow on $\mathrm{U}_{\mathrm{sym}}\left(\mathbb{C}^{n}\right)$ has the form $\partial u=\xi u-u \xi^{*}$ where $\xi: \mathbb{R} \times \mathrm{U}_{\mathrm{sym}}\left(\mathbb{C}^{n}\right) \rightarrow \mathfrak{u}\left(\mathbb{C}^{n}\right)$ is given by

$$
\xi:=D-\frac{1}{2}\left(u C^{*}-C u^{\dagger}\right) .
$$

We can pullback the flow $u: \mathbb{R} \rightarrow \mathrm{U}_{\text {sym }}\left(\mathbb{C}^{n}\right)$ to the flow $g: \mathbb{R} \rightarrow \mathrm{U}\left(\mathbb{C}^{n}\right)$ on the unitary group given by $\partial g=\xi g$. Hence we can pullback the flow $g: \mathbb{R} \rightarrow \mathrm{U}\left(\mathbb{C}^{n}\right)$ to the flow $\sigma: \mathbb{R} \rightarrow \mathfrak{u}\left(\mathbb{C}^{n}\right)$ on the unitary Lie algebra given by

$$
\partial \sigma=\operatorname{dexp}_{\sigma}^{-1} \circ \xi \text {. }
$$

Here $\operatorname{dexp} \bar{\sigma}^{-1}: \mathfrak{u}\left(\mathbb{C}^{n}\right) \rightarrow \mathfrak{u}\left(\mathbb{C}^{n}\right)$ is the operator $\operatorname{dexp}_{\sigma}^{-1}:=\operatorname{ad}_{\sigma} /\left(\exp \operatorname{ad}_{\sigma}-\mathrm{id}\right)$, where ad: $\mathfrak{u}\left(\mathbb{C}^{n}\right) \times \mathfrak{u}\left(\mathbb{C}^{n}\right) \rightarrow \mathfrak{u}\left(\mathbb{C}^{n}\right)$ is the Lie algebra commutator operator given in coordinates by $\operatorname{ad}_{\sigma} \circ \zeta:=[\sigma, \zeta]$ for any $\sigma, \zeta \in \mathfrak{u}\left(\mathbb{C}^{n}\right)$.

Proof. That the unitary symmetric flow on $\mathrm{U}_{\text {sym }}\left(\mathbb{C}^{n}\right)$ has the form $\partial u=\xi u-u \xi^{*}$ can be checked by inspection. This establishes the vector field governing the flow is a unitary Lie algebra action. The pullback of the flow via the Lie group action to the unitary Lie group $\mathrm{U}\left(\mathbb{C}^{n}\right)$ to the flow $\partial g=\xi g$ is then standard. The pullback of the unitary flow via the exponential map to the unitary Lie algebra $\mathfrak{u}\left(\mathbb{C}^{n}\right)$ to the flow stated is also standard; see Munthe-Kaas [21] or Malham and Wiese [19].

Corollary 5.3 (Trace of the Lie algebra flow). The angle $\theta \in \mathfrak{u}\left(\mathbb{C}^{1}\right)$ between the evolving Lagrangian plane and the standard reference plane is given by

$$
\theta=-2 \mathrm{i} \operatorname{tr} \sigma+\theta_{0},
$$

where $\theta_{0}$ is its initial value and $\operatorname{tr}: \mathfrak{u}\left(\mathbb{C}^{n}\right) \rightarrow \mathfrak{u}\left(\mathbb{C}^{1}\right)$ is the trace map.

Proof. Using the unitary Lie group action on $\mathrm{U}_{\text {sym }}\left(\mathbb{C}^{n}\right)$ we observe that

$$
\mathrm{e}^{\mathrm{i} \theta}=\operatorname{det} u=\operatorname{det} g u_{0} g^{\mathrm{T}}=(\operatorname{det} g)^{2} \operatorname{det} u_{0}=\exp (2 \operatorname{tr} \sigma) \operatorname{det} u_{0} .
$$

Taking the logarithm and setting $\theta_{0}=-\mathrm{i} \log \operatorname{det} u_{0}$ gives the result.

Remark 5.4. To compute the evolution of the angle between the evolving Lagrangian plane and the standard reference plane we must solve the differential equation for $\sigma$ in Theorem 5.2 and monitor its trace. Typically this procedure will be numerical. Fortunately, however, numerical procedures for solving for $\sigma$ (or $g$ or $u$ ) are robust in the sense that truncations of the vector field prescribing the evolution of $\sigma$, required for implementing Runge-Kutta methods for example, still lie in the unitary Lie algebra. Indeed this is the idea underlying Lie group numerical methods such as Runge-Kutta Munthe-Kaas methods developed by MuntheKaas [21. Similar approaches to those we propose here can be found in Maple and Marletta [20] and Greenberg and Marletta [12]. 

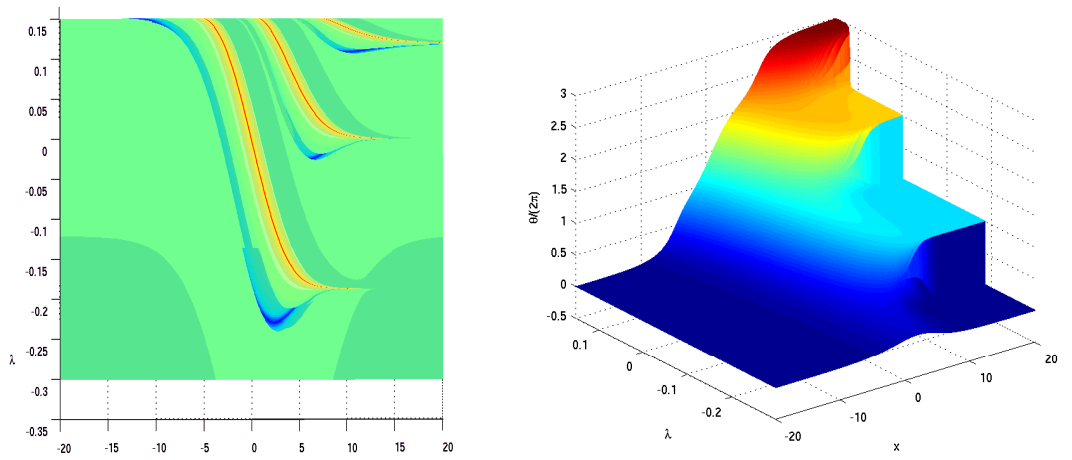

FIgURE 1. In the left panel, we plot the singular eigenvalue of $s=s(x, \lambda) \in \mathrm{D}\left(\mathbb{R}^{3}\right)$. The other two eigenvalues of $s$ are finite. Indeed for fixed values of $\lambda \in[-0.3,0.15]$ as shown, we integrate the Riccati equation for $s$ from $x=-20$ through to $x=20$ and record the behaviour of the eigenvalues of $s$. Red curves indicate the eigenvalue becoming infinite while the blue curves indicate the eigenvalue becoming zero. As expected, as $\lambda$ increases and passes through each spectral eigenvalue, the number of singularites in an eigenvalue of $s$ in the interval $[-20,20]$ jumps by an integer value. In the right panel, we plot the trace $\theta=\theta(x, \lambda)=-2 \mathrm{i} \operatorname{tr} \sigma(x, \lambda) \in \mathfrak{u}\left(\mathbb{C}^{1}\right)$ from Corollary 5.3 for $\lambda \in[-0.3,0.15]$ and $x \in[-20,20]$. As expected, as $\lambda$ increases and passes through each spectral eigenvalue, the value of $\theta(20, \lambda)$ jumps by an integer.

\section{EXAMPLE}

We apply our methods to the KdV equation from Chardard et al. [7 given by

$$
\partial_{t} U-c \partial_{x} U+U \partial_{x} U+\partial_{x}^{3} U-\partial_{x}^{5} U+\sigma \partial_{x}^{7} U=0
$$

where $c=71000 / 2159^{2}$ and $\sigma=0.2159$ are constants. This has a solitary-wave solution given by $U_{*}(x)=a\left(\operatorname{sech}^{6} k x+\operatorname{sech}^{4} k x\right)$ where $a=1039500 / 2159^{2}$ and $k=$ $(25 / 2159)^{1 / 2}$. The above equation is Hamiltonian, and the stability of the solitary wave can be determined by computing the spectrum of the Hessian of the Hamiltonian, i.e. we consider the selfadjoint spectral problem $\left(c-U_{*}-\partial_{x}^{2}+\partial_{x}^{4}-\sigma \partial_{x}^{6}\right) U=\lambda U$. Setting $(q, p)^{\mathrm{T}}:=\left(U, \partial_{x}^{2} U-\sigma \partial_{x}^{4} U, \partial_{x}^{2} U, \partial_{x} U-\partial_{x}^{3} U+\sigma \partial_{x}^{5} U,-\partial_{x} U, \sigma \partial_{x}^{3} U\right)^{\mathrm{T}}$ we find that our goal is to compute the values of the spectral parameter $\lambda \in \mathbb{R}$ for which the kernel of the operator $\partial-A$ is non-empty - we call these spectral eigenvalueswhere

$$
A(x, \lambda)=\left(\begin{array}{cccccc}
0 & 0 & 0 & 0 & -1 & 0 \\
0 & 0 & 0 & -1 & -1 & 0 \\
0 & 0 & 0 & 0 & 0 & 1 / \sigma \\
-\lambda+c-U_{*}(x) & 0 & 0 & 0 & 0 & 0 \\
0 & 0 & -1 & 0 & 0 & 0 \\
0 & -1 & 1 & 0 & 0 & 0
\end{array}\right) .
$$


See Chardard et al. [7] for details. For fixed values of $\lambda \in \mathbb{R}$ we evolve the unstable manifold, here the Lagrangian plane parameterized by $s=s(x, \lambda) \in \mathrm{D}\left(\mathbb{R}^{3}\right)$, from the $x \rightarrow-\infty$ far-field forward to $x \rightarrow+\infty$. We used the method of Schiff and Shnider [26] to integrate the Riccati equation through singularites; also see Ledoux et al. [18. We record the eigenvalues $\mu_{i}=\mu_{i}(x, \lambda), i=1,2,3$. Figure 1 (left panel) shows the eigenvalue of $s=s(x, \lambda)$ that becomes singular - the other two eigenvalues of $s$ remain finite for all $(x, \lambda) \in \mathbb{R}^{2}$. We see three curves in the $(x, \lambda)$ plane where the eigenvalue of $s$ becomes singular as we expect. Further for large $x$ the singular eigenvalue curves asymptotically approach fixed values - indicating an intersection of the evolved Lagrangian plane with the far-field Lagrangian plane and signifying a spectral eigenvalue. For fixed values of $\lambda \in \mathbb{R}$ we also evolved the Lagrangian plane parameterized by $\sigma=\sigma(x, \lambda) \in \mathfrak{u}\left(\mathbb{C}^{3}\right)$, i.e. by the flow on the unitary Lie algebra given in Theorem 5.2. This is the pullback of the flow on the manifold of unitary symmetric matrices via the Lie algebra action $\ell$. Explicitly this is

$$
\partial_{x} \sigma=\operatorname{dexp}_{\sigma}^{-1} \circ \xi\left(x, \exp \sigma u\left(x_{0}\right) \exp \sigma^{\mathrm{T}}\right),
$$

with data $\sigma\left(x_{0}\right)=O$ and where $u\left(x_{0}\right)$ is the symmetric unitary data. We chose a simple Euler method to numerically integrate this non-linear differential equation. This means for a fixed $\lambda$, for the data $u_{0}=u_{0}(\lambda)$ we set

$$
s_{0}(\lambda)=p(-\infty, \lambda) q^{-1}(-\infty, \lambda) \quad \text { and } \quad u_{0}(\lambda)=\left(\mathrm{id}_{3}-\mathrm{i} s_{0}(\lambda)\right)\left(\mathrm{id}_{3}+\mathrm{i} s_{0}(\lambda)\right)^{-1} .
$$

Euler integration for a sufficiently large number of timesteps $M$ and corresponding stepsize $h$ is given for $m=0,1,2, \ldots, M-1$ by

$$
\begin{aligned}
& \sigma_{m+1}=h\left(D\left(x_{m}, \lambda\right)-\frac{1}{2}\left(u_{m} C^{*}\left(x_{m}, \lambda\right)-C\left(x_{m}, \lambda\right) u_{m}^{\dagger}\right)\right), \\
& u_{m+1}=\exp \left(\sigma_{m+1}\right) \cdot u_{m} \cdot \exp \left(\sigma_{m+1}^{\mathrm{T}}\right) .
\end{aligned}
$$

The matrices $C=C(x, \lambda)$ and $D=D(x, \lambda)$ are explicitly defined in Lemma 5.1 . The real and imaginary parts of the components of $\sigma(x, 0.15)$ for $x \in[-20,20]$ are shown in Figure 2. As we expect, the solution components are well behaved and integration procedure very robust. In Figure 1 (right panel) we plot the trace $\theta=\theta(x, \lambda)=-2 \mathrm{i} \operatorname{tr} \sigma(x, \lambda) \in \mathfrak{u}\left(\mathbb{C}^{1}\right)$ from Corollary 5.3 for $\lambda \in[-0.3,0.15]$ and $x \in[-20,20]$. As $\lambda$ increases and passes through each spectral eigenvalue, the value of $\theta$ jumps by an integer, contributing to the Maslov index.

\section{Concluding Remarks}

What about the infinite dimensional case? Consider a formal example to give the context and help clarify the picture. Recall the reaction-diffusion parabolic partial differential equation in the introduction. However now suppose it admits travellingwave solutions on $\mathbb{R} \times \mathbb{T}$. Here we suppose the waves travel in the direction along $\mathbb{R}$ and we have transverse periodic boundary conditions indicated by the 1-dimensional torus $\mathbb{T}$ (our subsequent formal analysis also applies to higher dimensional tori). The linear stability of such waves is determined by the structure of the spectrum of an unbounded Fredholm operator of the form

$$
H:=B\left(\partial_{x}^{2}+\Delta_{y}\right)+d \partial_{x}+V
$$

for $x \in \mathbb{R}$ and $y \in \mathbb{T}$. The $n \times n$ matrix $B$ is unchanged from our original example, the matrix $d=d(x)$ represents convection and the $n \times n$ potential matrix $V=$ $V(x, y)$. We assume $V(x, y) \rightarrow V_{ \pm}(y)$, as $x \rightarrow \pm \infty$. Of interest is the eigenvalue 

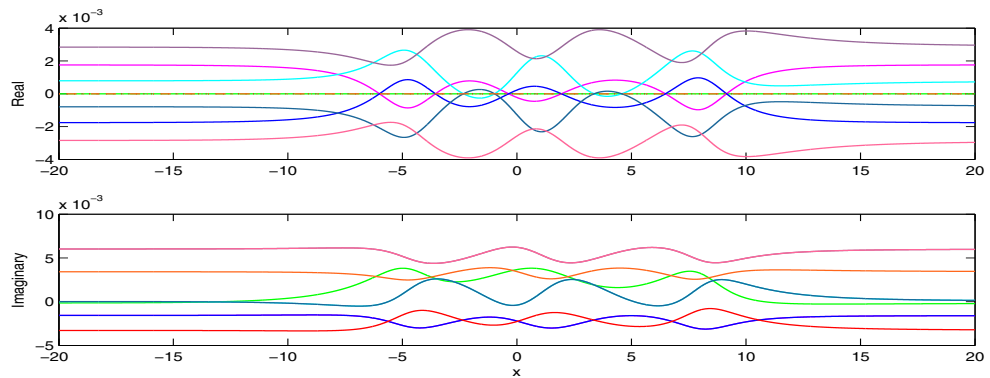

FiguRE 2. The panels show the real (top) and imaginary (bottom) parts of the components of the flow on the unitary Lie algebra, i.e. for $\sigma=\sigma(x, \lambda) \in \mathfrak{u}\left(\mathbb{C}^{3}\right)$ for $\lambda=0.15$ and $x \in[-20,20]$; see Theorem 5.2. Since $\sigma$ is anti-Hermitian there are six non-zero real parts and six distinct imaginary parts (three are repeated).

problem $H q=\lambda q$. We re-write the eigenvalue problem as follows. We represent the solution $q$ using a transverse Fourier-Galerkin basis $\left\{\varphi_{k}(y): k \in \mathbb{Z}\right\}$ where $\Delta_{y} \varphi_{k}=-|k|^{2} \varphi_{k}$. Our eigenvalue problem can thus be viewed as an eigenvalue problem in $L^{2}\left(\mathbb{R} ; L^{2}\left(\mathbb{T} ; \mathbb{C}^{n}\right)\right)$. Since $L^{2}\left(\mathbb{T} ; \mathbb{C}^{n}\right) \cong\left(\ell^{2}\right)^{n}$, where $\ell^{2}$ denotes the usual Hilbert sequence space, we identify each element $q=q(x)$ in $L^{2}\left(\mathbb{T} ; \mathbb{C}^{n}\right)$ with a sequence $\hat{q}:=\left\{\hat{q}_{k}: k \in \mathbb{Z}\right\}$ in $\left(\ell^{2}\right)^{\otimes n}$, i.e. for each $x \in \mathbb{R}$ we replace $q(x)$ by $\hat{q}(x)$. Then in this example our separable Hilbert space is $\mathbb{H}=L^{2}\left(\mathbb{T} ; \mathbb{C}^{2 n}\right)$ and our phase space $L^{2}(\mathbb{R} ; \mathbb{H})$. Our eigenvalue problem above now has the form $\partial^{2} \hat{q}=d \partial \hat{q}+c \hat{q}$ where we replace $-B^{-1} d$ by $d$. However here we define

$$
c: \hat{q} \mapsto B^{-1}\left(\lambda \hat{q}+K^{2} \hat{q}-V \star \hat{q}\right)
$$

where $K^{2}$ is the linear diagonal operator on $\left(\ell^{2}\right)^{\otimes n}$ that sends each component $\hat{q}_{k}$ of $\hat{q}$ to $|k|^{2} \hat{q}_{k}$ and $V \star \hat{q}$ is the usual discrete convolution product in $\left(\ell^{2}\right)^{\otimes n}$. If we now set $\hat{p}:=\partial \hat{q}$, then our eigenvalue problem in $L^{2}(\mathbb{R} ; \mathbb{H})$ is

$$
(\partial-A)\left(\begin{array}{c}
\hat{q} \\
\hat{p}
\end{array}\right)=O
$$

where $A=A(x ; \lambda)$ has the usual block form with $a=O, b=\mathrm{id}$ and $c$ and $d$ are as defined above. Formally we can still define a compact map $s: \hat{q} \rightarrow \hat{p}$ and $s$ satifies the Riccati equation $\partial s=c+d s-s(a+b s)$. Let $s_{0}$ denote the map $s_{0}: \hat{q}(-\infty) \rightarrow \hat{p}(-\infty)$. Then $s_{0}$ satisfies $c_{0}+d s_{0}-s_{0}\left(a+b s_{0}\right)=O$, where $c_{0}=c(-\infty)$. Setting $s=s_{0}+\hat{s}$ and $c=c_{0}+\hat{c}$, then $\hat{s}$ satisfies the Riccati equation

$$
\partial \hat{s}=\hat{c}+\left(d-s_{0} b\right) \hat{s}-\hat{s}\left(a+b s_{0}+b \hat{s}\right) .
$$

Note the unbounded operator $K^{2}$ present in $c$ is not present in this Riccati equation.

Suppose $\mathbb{H}$ is a separable Hilbert space with a $\mathbb{Z}_{2}$-grading $\mathbb{H}=\mathbb{Q} \oplus \mathbb{P}$. Let $G L(\mathbb{H})$ denote the group of bounded invertible operators on $\mathbb{H}$. We define $\mathrm{GL}_{\text {res }}(\mathbb{H})$ to be the subgroup of GL(H) whose elements 'preserve' the $\mathbb{Z}_{2}$-grading of $\mathbb{H}$; see Pressley and Segal $\left[22\right.$. Indeed for each element of $\mathrm{GL}_{\mathrm{res}}(\mathbb{H})$, the diagonal maps $\mathbb{Q} \rightarrow \mathbb{Q}$ and $\mathbb{P} \rightarrow \mathbb{P}$ are Fredholm while the off-diagonal maps $s: \mathbb{Q} \rightarrow \mathbb{P}$ and $r: \mathbb{P} \rightarrow \mathbb{Q}$ are Hilbert-Schmidt. Indeed the map $s$ parameterizes a chart of the Fredholm Grassmannian. In the symplectic case ( $V$ is selfadjoint and $d=0$ above), the 
Hilbert space $\mathbb{H}$ is symplectic and subspaces $\mathbb{Q} \subset \mathbb{H}$ Lagrangian. The Lagrangian Grassmannian $\Lambda(\mathbb{H})$ is the space of Lagrangian subspaces. Its fundamental group $\pi_{1}(\Lambda(\mathbb{H}))$ is trivial. However following Furutani [11] the subset of $\Lambda(\mathbb{H})$ parameterized by $s$, the Fredholm Lagrangian Grassmannian, has fundamental group $\mathbb{Z}$. Further, the determinant of the Cayley map of $s$ is well defined via Fredholm (or modified) determinants. This is the direction we intend to pursue next.

\section{ACKNOWLEDGEMENTS}

The authors thank Graham Cox, Chris Jones, Yuri Latushkin, Veerle Ledoux and Robbie Marangell for helpful discussions. The first author would like to thank the Institute for Mathematics and its Applications at the University of Minnesota where part of this work was conducted.

\section{REFERENCES}

[1] J. Alexander, R. Gardner, and C. Jones, A topological invariant arising in the stability analysis of travelling waves, J. Reine Angew. Math. 410 (1990), 167-212. MR1068805|(92d:58028)

[2] A.Y. Aljasser, Computing the Evans function for the stability of combustion waves, PhD thesis, 2012.

[3] V. I. Arnol'd, On a characteristic class entering into conditions of quantization (Russian), Funkcional. Anal. i Priložen. 1 (1967), 1-14. MR0211415 (35 \#2296)

[4] V. I. Arnol'd, Sturm theorems and symplectic geometry (Russian), Funktsional. Anal. i Prilozhen. 19 (1985), no. 4, 1-10, 95. MR820079 (87j:58033)

[5] Amitabha Bose and Christopher K. R. T. Jones, Stability of the in-phase travelling wave solution in a pair of coupled nerve fibers, Indiana Univ. Math. J. 44 (1995), no. 1, 189-220, DOI 10.1512/iumj.1995.44.1984. MR.1336438(96k:92005)

[6] Jonathan Bougie, Asim Gangopadhyaya, Jeffry Mallow, and Constantin Rasinariu, Supersymmetric quantum mechanics and solvable models, Symmetry 4 (2012), no. 3, 452-473, DOI 10.3390/sym4030452. MR.2979407

[7] Frédéric Chardard, Frédéric Dias, and Thomas J. Bridges, Computing the Maslov index of solitary waves, Part 2: Phase space with dimension greater than four, Phys. D 240 (2011), no. 17, 1334-1344, DOI 10.1016/j.physd.2011.05.014. MR2831770 (2012k:37130)

[8] M. A. de Gosson, The principles of Newtonian and quantum mechanics, Imperial College Press, London, 2001. The need for Planck's constant, $h$; With a foreword by Basil Hiley. MR1897416 (2003k:81004)

[9] Jian Deng and Christopher Jones, Multi-dimensional Morse index theorems and a symplectic view of elliptic boundary value problems, Trans. Amer. Math. Soc. 363 (2011), no. 3, 14871508, DOI 10.1090/S0002-9947-2010-05129-3. MR2737274(2011k:35156)

[10] Jian Deng and Shunsau Nii, An infinite-dimensional Evans function theory for elliptic boundary value problems, J. Differential Equations 244 (2008), no. 4, 753-765, DOI 10.1016/j.jde.2007.10.037. MR.2391343 (2009a:35180)

[11] Kenro Furutani, Fredholm-Lagrangian-Grassmannian and the Maslov index, J. Geom. Phys. 51 (2004), no. 3, 269-331, DOI 10.1016/j.geomphys.2004.04.001. MR2079414 (2005g:53150)

[12] Leon Greenberg and Marco Marletta, Numerical methods for higher order Sturm-Liouville problems, Numerical analysis 2000, Vol. VI, Ordinary differential equations and integral equations, J. Comput. Appl. Math. 125 (2000), no. 1-2, 367-383, DOI 10.1016/S03770427(00)00480-5. MR 1803203(2001k:65123)

[13] Jeffrey Humpherys and Kevin Zumbrun, An efficient shooting algorithm for Evans function calculations in large systems, Phys. D 220 (2006), no. 2, 116-126, DOI 10.1016/j.physd.2006.07.003. MR2253406 (2007e:35006)

[14] Christopher K. R. T. Jones, Yuri Latushkin, and Robert Marangell, The Morse and Maslov indices for matrix Hill's equations, Spectral analysis, differential equations and mathematical physics: a festschrift in honor of Fritz Gesztesy's 60th birthday, Proc. Sympos. Pure Math., vol. 87, Amer. Math. Soc., Providence, RI, 2013, pp. 205-233. MR3087908 
[15] Christopher K. R. T. Jones, Instability of standing waves for nonlinear Schrödinger-type equations, Ergodic Theory Dynam. Systems 8* (1988), Charles Conley Memorial Issue, 119138, DOI 10.1017/S014338570000938X. MR967634 (90d:35267)

[16] V. Ledoux and S.J.A. Malham, Spectral shooting is Schubert calculus, working paper 2009.

[17] Veerle Ledoux, Simon J. A. Malham, Jitse Niesen, and Vera Thümmler, Computing stability of multidimensional traveling waves, SIAM J. Appl. Dyn. Syst. 8 (2009), no. 1, 480-507, DOI 10.1137/080724009. MR2496765 (2010a:65127)

[18] Veerle Ledoux, Simon J. A. Malham, and Vera Thümmler, Grassmannian spectral shooting, Math. Comp. 79 (2010), no. 271, 1585-1619, DOI 10.1090/S0025-5718-10-02323-9. MR2630004 (2011c:65139)

[19] Simon J. A. Malham and Anke Wiese, Stochastic Lie group integrators, SIAM J. Sci. Comput. 30 (2008), no. 2, 597-617, DOI 10.1137/060666743. MR2385877(2009b:37154)

[20] Carsten R. Maple and Marco Marletta, Solving Hamiltonian systems arising from ODE eigenproblems, Numer. Algorithms 22 (1999), no. 3-4, 263-284 (2000), DOI 10.1023/A:1019171110743. MR:1749487(2001c:65158)

[21] Hans Munthe-Kaas, High order Runge-Kutta methods on manifolds, Proceedings of the NSF/CBMS Regional Conference on Numerical Analysis of Hamiltonian Differential Equations (Golden, CO, 1997), Appl. Numer. Math. 29 (1999), no. 1, 115-127, DOI 10.1016/S01689274(98)00030-0. MR 1662814 (99i:65075)

[22] Andrew Pressley and Graeme Segal, Loop groups, Oxford Mathematical Monographs, The Clarendon Press, Oxford University Press, New York, 1986. Oxford Science Publications. MR.900587 (88i:22049)

[23] Joel Robbin and Dietmar Salamon, The spectral flow and the Maslov index, Bull. London Math. Soc. 27 (1995), no. 1, 1-33, DOI 10.1112/blms/27.1.1. MR.1331677 (96d:58021)

[24] Björn Sandstede, Stability of travelling waves, Handbook of dynamical systems, Vol. 2, North-Holland, Amsterdam, 2002, pp. 983-1055, DOI 10.1016/S1874-575X(02)80039-X. MR.1901069 (2004e:37121)

[25] Myunghyun $\mathrm{Oh}$ and Björn Sandstede, Evans functions for periodic waves on infinite cylindrical domains, J. Differential Equations 248 (2010), no. 3, 544-555, DOI 10.1016/j.jde.2009.08.003. MR2557905 (2011d:35257)

[26] Jeremy Schiff and S. Shnider, A natural approach to the numerical integration of Riccati differential equations, SIAM J. Numer. Anal. 36 (1999), no. 5, 1392-1413 (electronic), DOI 10.1137/S0036142996307946. MR.1706774 (2000d:34024)

Maxwell Institute for Mathematical Sciences and School of Mathematical and Computer Sciences, Heriot-Watt University, Edinburgh EH14 4AS, United Kingdom; Department of Mathematics, Boston University, Boston, Massachusetts 02215

E-mail address: mabeck@math.bu.edu

Maxwell Institute for Mathematical Sciences and School of Mathematical and ComPuter Sciences, Heriot-Watt University, Edinburgh EH14 4AS, United Kingdom

E-mail address: S.J.Malham@ma.hw.ac.uk 